TecnoLógicas

ISSN-p 0123-7799

ISSN-e 2256-5337

Vol. 21, No. 41, pp. 45-62

Enero-abril de 2018

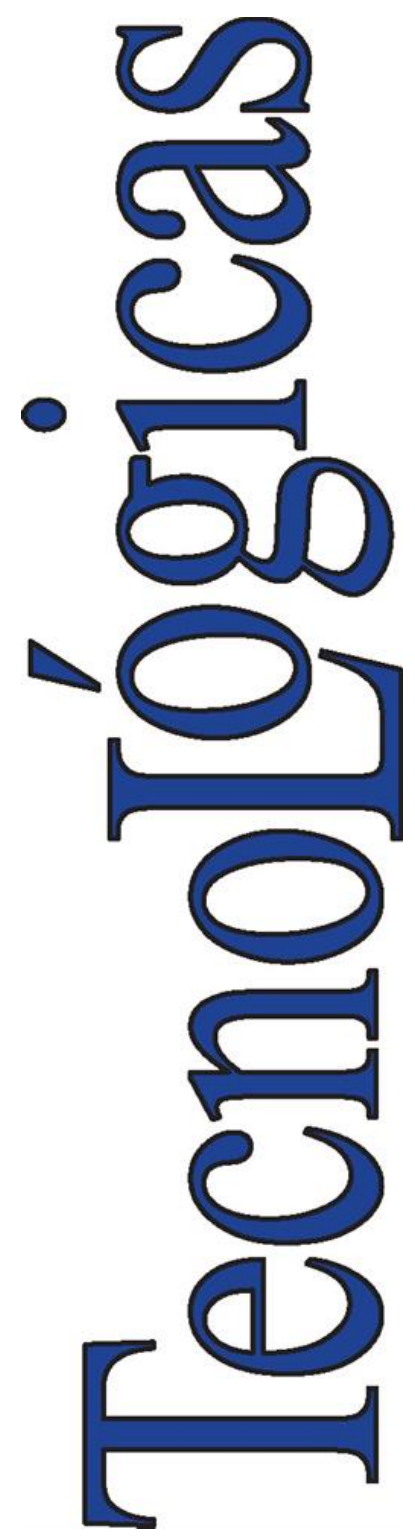

(C) Copyright 2015 por autores y Tecno Lógicas Este trabajo está licenciado bajo una Licencia Internacional Creative Commons Atribución (CC BY)

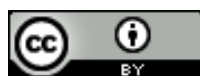

Artículo de Investigación/Research Article

\section{Análisis comparativo del control predictivo de corriente en convertidores VSI empleados en la conexión a red de energías renovables}

\section{Comparative analysis of predictive current control of VSI converters in the connection of renewable energy sources to the power grid}

\author{
Duberney Murillo-Yarce ${ }^{1}$, Alfonso Alzate-Gómez ${ }^{2}$ \\ y Andrés Escobar-Mejía ${ }^{3}$
}

Recibido: 13 de octubre de 2017

Aceptado: 30 de noviembre de 2017

Cómo citar / How to cite

D. Murillo-Yarce, A. Alzate-Gómez, y A. Escobar-Mejía, Análisis comparativo del control predictivo de corriente en convertidores VSI empleados en la conexión a red de energías renovables. TecnoLógicas, vol. 21, no. 41, pp. 45-62, 2018.

1 MSc en Ingeniería Eléctrica, Programa Ingeniería Eléctrica, Universidad Tecnológica de Pereira, Pereira-Colombia, duberm@utp.edu.co

2 MSc en Ingeniería Eléctrica, Programa Ingeniería Eléctrica, Universidad Tecnológica de Pereira, Pereira-Colombia, alalzate@utp.edu.co

$3 \mathrm{PhD}$. en Ingeniería Eléctrica, Programa Ingeniería Eléctrica, Universidad Tecnológica de Pereira, andreses1@utp.edu.co 


\title{
Resumen
}

Este artículo analiza el desempeño del control de corriente predictivo en la operación de inversores fuente de voltaje o Voltage Source Inverter (VSI), que sirven de elemento de conexión de energías renovables a la red eléctrica. El VSI es comúnmente utilizado para convertir señales de corriente continua, obtenidas a partir de fuentes renovables o de sistemas de acumulación, en señales de corriente alterna de amplitud y frecuencia constantes. Con este propósito, se implementa una estrategia que define la secuencia de conmutación de los semiconductores de potencia del convertidor conocida como técnica PWM. Además, se requiere realizar el control de la corriente que se inyecta a la red requiriendo un algoritmo de control adicional, que actúa en conjunto con la técnica PWM. El principal aporte del artículo consiste en demostrar que el control de corriente predictivo presenta mejores características en comparación con el control orientado de corriente, que es la estrategia más conocida. Inicialmente, se analizan las técnicas PWM convencionales y luego, se realiza la formulación de las técnicas de control de corriente. El control predictivo minimiza el error futuro de la corriente definiendo el mejor estado de conmutación en cada período. Las dos técnicas de control de corriente presentan buenos resultados. Pero sobresale el control predictivo por su simplicidad, fácil implementación, bajo contenido armónico y menor tiempo de respuesta.

\section{Palabras clave}

Convertidor fuente de tensión, técnica PWM, control de corriente, control orientado, control predictivo.

\begin{abstract}
This paper analyzes the performance of the predictive current control technique in the operation of Voltage Source Inverters (VSI) used to connect renewable resources to the power grid. VSIs are commonly used to convert DC power from renewable energy sources, such as energy storage units, solar power plants and fuel cells, to AC power with constant magnitude and frequency. For this purpose, a strategy known as PWM was implemented to determine the switching sequence of the converter's power semiconductors. In addition, the current being injected into the grid must be controlled; thus, an additional control algorithm was required to operate in conjunction with the PWM technique. The main contribution of this paper is demonstrating that predictive current control presents better characteristics than current-oriented control, the most widely used strategy. The first section analyzes conventional PWM techniques and, in the second part, current control techniques are formulated. Predictive control minimizes the future error of the current by defining the best switching state in each period. The two current control techniques present good performance. However, predictive control is preferable due to its simplicity, easy implementation, low harmonic content, as well as reduced response time.
\end{abstract}

\section{Keywords}

Voltage-source converter, PWM technique, Current control, Oriented control, Predictive control. 


\section{INTRODUCCIÓN}

El creciente interés por reducir la dependencia de fuentes de generación que emiten gases de efecto invernadero y la necesidad de consolidar escenarios favorables para el avance de las energías renovables, han impulsado el desarrollo e implementación de nuevos esquemas de generación como las plantas solares y eólicas, que a diferencia de las fuentes convencionales, requieren de un elemento acondicionador (que basa su operación en electrónica de potencia), el cual permite el acople con la red eléctrica de manera segura [1]. El elemento acondicionador es un conversor de energía denominado inversor fuente de voltaje o Voltage Source Inverter (VSI), el cual transforma la corriente y el voltaje de naturaleza fluctuante (comúnmente visto en sistemas eólicos y fotovoltaicos) en señales AC de magnitud constante y operando a la frecuencia de la red eléctrica.

Además de controlar amplitud y frecuencia, se requiere el control de la corriente que se inyecta a la red. La regulación de corriente es una acción de control ampliamente utilizada en fuentes renovables, pero también se aplica en sistemas de conversión de energía como accionamientos eléctricos, control de potencia reactiva, UPS y filtros activos [2].

El control de corriente compara las corrientes sensadas con las corrientes de referencia para generar la señal de control, que sirve como entrada a la modulación por ancho de pulso o Pulse-Width Modulation (PWM) responsable del encendido $\mathrm{y}$ apagado de los dispositivos semiconductores que conforman el inversor.

Una de las estrategias de control de corriente utilizada en inversores es el control orientado. Esta estrategia es una aplicación del control orientado de campo, que por mucho tiempo fue la estrategia de control generalizada en aplicaciones de máquinas de corriente continua conectadas a convertidores conmutados [3]. El control orientado de campo aplicado en máquinas eléctricas DC regulaba la corriente de armadura. Esta estrategia de control se utilizó después para el control de voltaje y corriente en inversores de potencia, redefiniéndose como control orientado. La primera aplicación del control orientado en inversores se expuso en [4].

Luego, aparece el control predictivo como una nueva tendencia de control en el área de accionamientos eléctricos [5]. Este controlador presentaba una estructura intuitiva y de simple implementación, que posteriormente se extendió a filtros activos, rectificadores e inversores [6]. Hoy en día, el control predictivo de corriente es uno de los algoritmos más populares y más efectivos en inversores considerando su rápida respuesta, cero errores de estado estacionario, compensación del tiempo de retardo y completa compatibilidad para su implementación en sistemas digitales [7]. En la literatura se encuentran aplicaciones de control predictivo de corriente en diferentes tipos de inversores VSI: dos niveles (o simplemente inversor VSI) [8], [14], inversor multinivel [15], [17] y convertidores modulares multinivel [18], [19].

En el caso de inversores VSI, las primeras aplicaciones de control predictivo de corriente planteaban soluciones de buen comportamiento y fácil implementación [8]. Los controladores diseñados posteriormente, presentaban ventajas comparativas respecto al controlador clásico. En [9] se presenta un controlador que opera con frecuencia de conmutación constante. En [10] se mejora el desempeño de estado estacionario de un convertidor aislado disminuyendo el tiempo de muestreo. En [11] el control predictivo realiza la compensación de fase para disminuir el rizado de la corriente. Inicialmente la investigación se orientó a sistemas sin conexión a red y luego, a sistemas interconectados. Diferentes controladores han sido desarrollados a partir del concepto original para mejorar la respuesta del inversor en sistemas con conexión a red. En [12], el control predictivo es mejorado utilizando estimación pa- 
ramétrica, mientras que en [13] se desarrolla un control predictivo robusto de corriente. Por su parte, en [14] se diseña un filtro para disminuir la distorsión armónica de la corriente inyectada en el punto de conexión.

En inversores multinivel y convertidores modulares, las investigaciones se orientan al mejoramiento de la respuesta dinámica. En [15] se presenta un control de corriente predictivo para un inversor VSI de tres niveles alimentando una carga y en [16] se presenta un control predictivo para un inversor de cinco niveles con tres variables de control: voltaje del capacitor, corriente de entrada y de salida. En [17], el controlador predictivo actúa en un inversor multinivel asimétrico en cascada con condensador flotante conectado a la red incluyendo resultados experimentales para un inversor de siete niveles. El objetivo de las investigaciones en el control predictivo de corriente en convertidores modulares multinivel es la reducción del cálculo computacional tal como se describe en [18],[19].

En la actualidad, crece el interés y las aplicaciones del control de corriente predictivo en inversores, convertidores y accionamientos eléctricos. Este artículo de investigación se enfoca en la aplicación del control predictivo de corriente en inversores conectados a la red. El controlador predictivo presenta ventajas respecto al control orientado, pero estas ventajas no han sido cuantificadas en otros trabajos de investigación. El aporte principal de este trabajo es realizar un análisis comparativo entre el control de corriente predictivo que es la tendencia actual y el control orientado de corriente que es la estrategia clásica.

Este artículo está organizado en cuatro secciones. La sección dos presenta la metodología. Inicialmente se presenta la estructura del inversor VSI y el análisis de las técnicas PWM. Luego, se exponen los controladores de corriente (control orientado y control predictivo). En la sección tres, se muestra el análisis comparativo de los dos controladores, considerando la distorsión armónica, el comportamiento dinámico, la conmutación de los dispositivos y el tiempo de muestreo. Finalmente, se presentan las conclusiones de la investigación en la sección cuatro.

\section{METODOLOGíA}

\subsection{Estructura VSI}

El inversor VSI está compuesto por un condensador, un conjunto de dispositivos semiconductores y un filtro. El condensador es el elemento almacenador de energía. Los semiconductores son los elementos que mediante su conmutación sintetizan las señales de referencia que son inyectadas a la red en el punto de conexión. El filtro atenúa el efecto armónico consecuencia de la conmutación del convertidor. Fig. 1 muestra el inversor VSI de tres ramas y sus componentes, en una conexión a red.

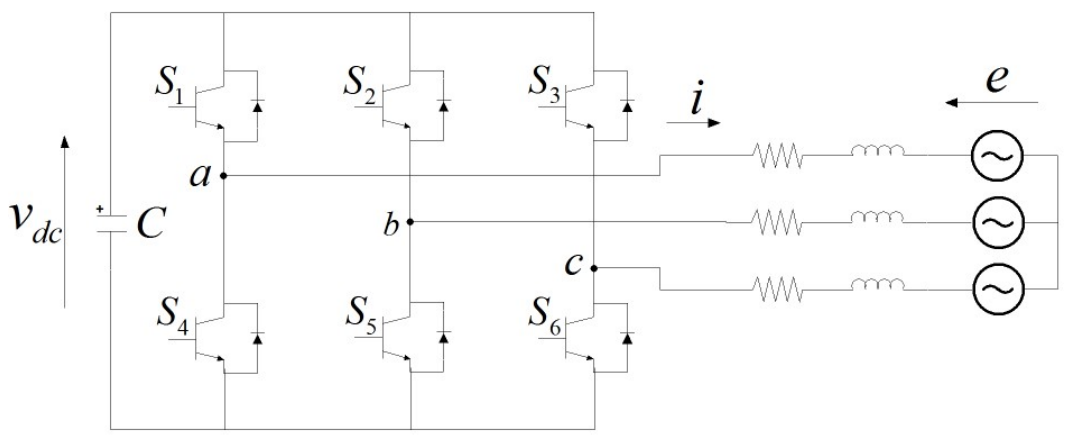

Fig. 1. Convertidor VSI de tres ramas conectado a red. Fuente: autores. 


\subsection{Técnicas PWM}

Las técnicas PWM se pueden clasificar en dos grupos principales: modulación basada en portadora y modulación vectorial. La modulación basada en portadora es simple, presenta bajo tiempo computacional y fácil implementación [20]. La modulación vectorial presenta mejor desempeño: menor contenido armónico, secuencias de conmutación óptimas y mayores voltajes [21]. En esta técnica se obtiene un mayor aprovechamiento del voltaje del bus DC, como se demostró en [22]. En la modulación basada en portadora las señales de conmutación por fase son independientes, mientras en la modulación vectorial las señales de las tres fases están integradas en el mismo algoritmo [23].

La técnica más utilizada en la modulación basada en portadora es la modulación sinusoidal PWM (SPWM). En esta técnica la señal de referencia de naturaleza sinusoidal es comparada con una señal triangular de alta frecuencia. Así, las señales PWM obtenidas para cada rama guardan en forma implícita la frecuencia y la forma de la señal de referencia. En la modulación vectorial PWM (SVPWM), se obtiene una secuencia de conmutación asociada a vectores adyacentes según la posición del fasor del voltaje de referencia. Dependiendo de la simetría de las señales y del cambio de estado de los dispositivos, esta modulación puede ser continua o discontinua.
En la modulación SPWM la señal sinusoidal de frecuencia constante (moduladora) se compara con una señal triangular de mayor frecuencia (portadora), para definir las señales de conmutación de dispositivos de la misma rama que generan la señal de referencia de una fase. Para obtener las referencias de las otras dos fases, se repite el proceso considerando señales de la misma amplitud y desfasadas 120 grados. Una relación importante es el índice de modulación que relaciona la amplitud de la moduladora y la portadora, Fig. 2.

La modulación SVPWM, se basa en los vectores de conmutación que son los posibles estados de los dispositivos del puente inversor. El vector está formado por tres dígitos binarios, uno asociado a cada rama. Por ejemplo, el vector (110) implica que el dispositivo superior de la primera rama está activado (S1 ON), lo mismo que el dispositivo superior de la segunda rama (S2 ON), mientras que el dispositivo superior de la tercera rama está desactivado (S3 OFF). El estado de los dispositivos inferiores es contrario al del dispositivo superior, para evitar cortocircuitar el elemento almacenador de energía. Con los posibles estados de conmutación se obtiene el espacio vectorial definido en el plano complejo $\alpha \beta$, donde se ubica el vector voltaje de referencia. En Fig. 3 se observa el espacio vectorial y en la Tabla 1, la correspondencia de los vectores de conmutación en coordenadas $\alpha \beta$.

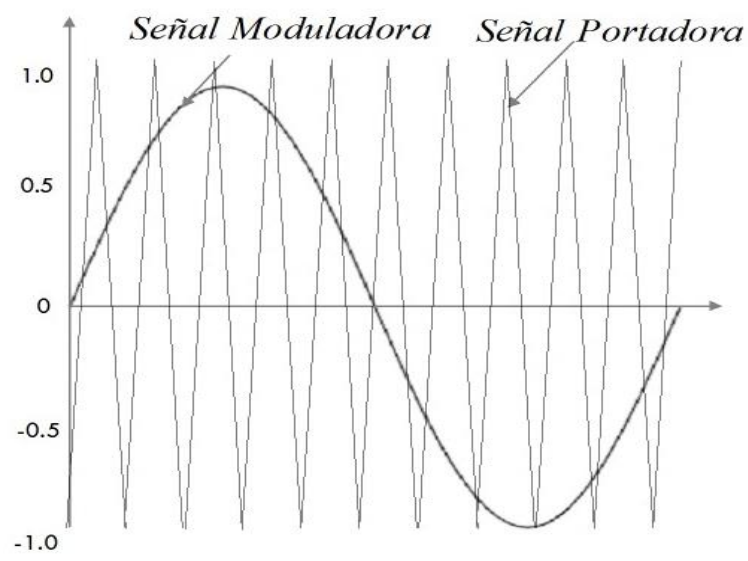

Fig. 2. Modulación SPWM monofásica. Fuente: autores. 


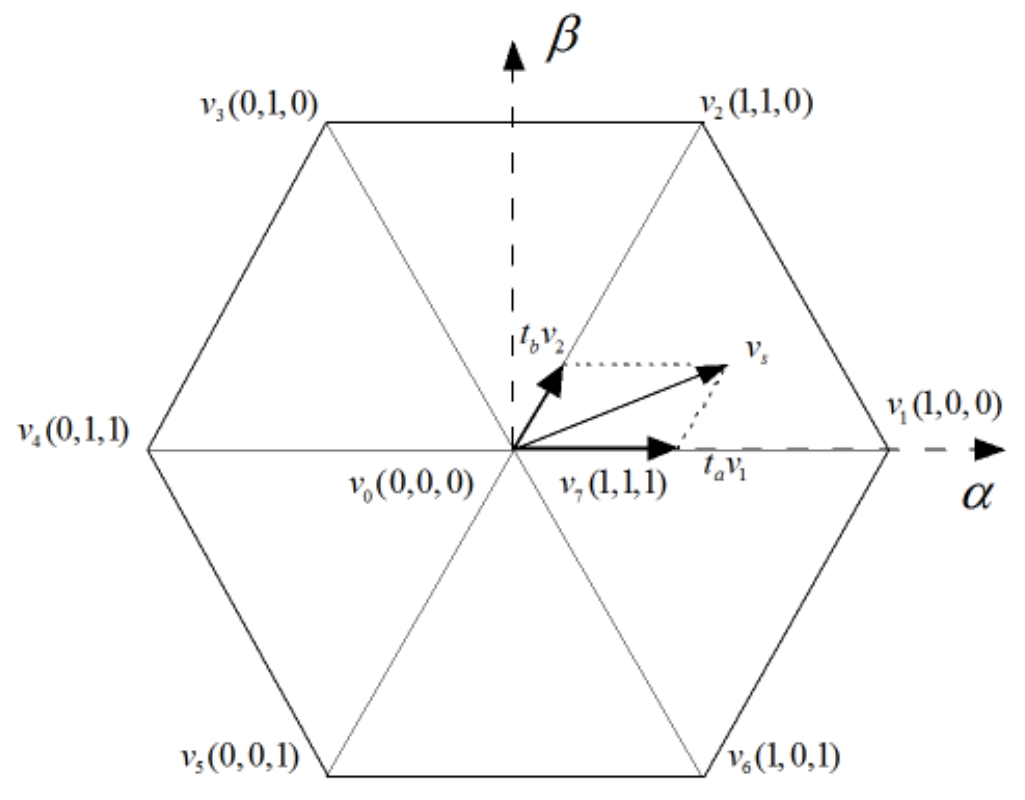

Fig. 3. Modulación SVPWM (sextantes). Fuente: autores.

Tabla 1. Vectores de conmutación en coordenadas $\alpha \beta$. Fuente: autores.

\begin{tabular}{ccccc}
\hline $\boldsymbol{v}$ & $\boldsymbol{S}_{\mathbf{1}}$ & $\boldsymbol{S}_{\mathbf{2}}$ & $\boldsymbol{S}_{\mathbf{3}}$ & $\boldsymbol{v}_{\boldsymbol{\alpha} \boldsymbol{\beta}}$ \\
\hline$v_{0}$ & 0 & 0 & 0 & 0 \\
$v_{1}$ & 1 & 0 & 0 & $2 / 3 V d c$ \\
$v_{2}$ & 1 & 1 & 0 & $(1 / 3+j \sqrt{3} / 3) V d c$ \\
$v_{3}$ & 0 & 1 & 0 & $(-1 / 3+j \sqrt{3} / 3) V d c$ \\
$v_{4}$ & 1 & 0 & 0 & $-2 / 3 V d c$ \\
$v_{5}$ & 0 & 0 & 1 & $(-1 / 3-j \sqrt{3} / 3) V d c$ \\
$v_{6}$ & 1 & 0 & 1 & $(1 / 3-j \sqrt{3} / 3) V d c$ \\
$v_{7}$ & 1 & 1 & 1 & 0 \\
\hline
\end{tabular}

La modulación SPWM sigue tres pasos fundamentales: selección de vectores, tiempos de aplicación y diseño de la secuencia en el período de conmutación [24]. La posición del vector de referencia se evalúa en cada período de muestreo, precisando los vectores de conmutación activos, que con los vectores nulos y los tiempos de conducción correspondientes, finalmente definen la secuencia de conmutación para el próximo período. Existen varias secuencias de conmutación posibles que se justifi- can por la libertad de distribuir el tiempo de aplicación del vector nulo entre los vectores nulos $v_{0} \mathrm{y} v_{7}$, y por los diferentes órdenes posibles para la aplicación de los vectores en un mismo período de conmutación.

En Fig. 3, se observa cómo el vector de referencia $v_{s}$ se descompone en los vectores adyacentes $v_{1}$ y $v_{2}$. El vector $v_{1}$ actúa durante un tiempo $t_{a} \mathrm{y}$ el vector $v_{2}$ durante el tiempo $t_{b}$ para sintetizar el vector $v_{s}$. El vector nulo $\left(v_{0}\right.$ ó $\left.v_{7}\right)$ actúa durante el tiem- 
po $t_{0}$ que corresponde al intervalo faltante del período de conmutación $T_{S},\left(t_{0}=T_{s}-\right.$ $\left.t_{a}-t_{b}\right)$.

Según el método de cálculo de la secuencia de conmutación, la técnica SVPWM se puede clasificar como continua o discontinua. La diferencia consiste en que en la modulación discontinua una de las ramas del inversor no se controla durante el período de conmutación, mientras que en la modulación continua sí hay control sobre cada rama en cada intervalo. La modulación discontinua tiene la ventaja de disminuir las pérdidas de conmutación, pero incrementa el contenido armónico de las señales de salida. Después de seleccionar entre conmutación continua o discontinua, aún queda pendiente definir el orden de aplicación de los vectores. Se utilizan secuencias centradas en el intervalo de conmutación para minimizar pérdidas. En Fig. 4, se describe una secuencia de conmutación continua y una secuencia discontinua, válidas para el vector de referencia $v_{s}$ mostrado en Fig. 3. En la secuencia discontinua se usó el vector nulo $v_{7}$. Una señal de conmutación diferente se hubiera obtenido con el vector nulo $v_{0}$. Ambas secuencias son factibles.

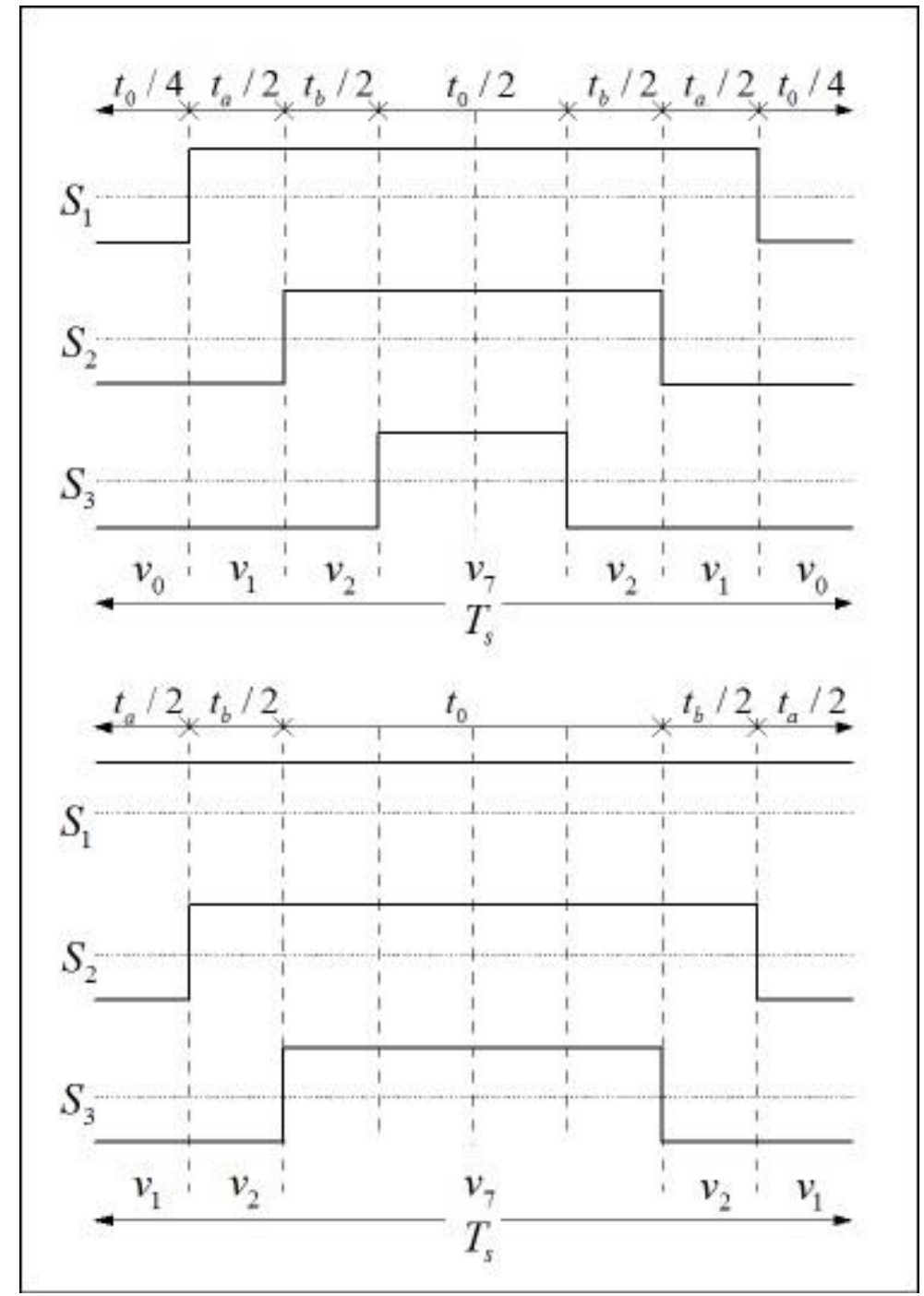

Fig. 4. Secuencia de conmutación técnica SVPWM: a) Continuo, b) Discontinuo. Fuente: autores. 


\subsection{Control orientado}

Una técnica de control de corriente es la aplicación del control orientado en el marco de referencia dq. En su implementación se requiere un lazo de enganche de fase $o$ Phase Locked Loop (PLL) que determina la posición angular, valor necesario para aplicar la transformación de coordenadas abc/dq y dq/abc. Un análisis detallado de esta transformación y su aplicación en control se expone en [25], [26].

Para implementar el controlador se requiere el modelo del sistema en coordenadas dq. En (1), se describe el convertidor VSI de la Fig. 1. en cantidades trifásicas, siendo $\mathrm{v}$ el voltaje de las ramas del inversor respecto a la referencia de la red. Aplicando transformación de coordenadas abc/ $\alpha \beta$ se obtiene (2) y mediante la relación (3) se llega al modelo del sistema en coordenadas dq indicado en (4).

$\mathbf{v}(\mathbf{t})=\mathbf{R i}(\mathbf{t})+\mathbf{L} \frac{\mathbf{d i}(\mathbf{t})}{\mathbf{d t}}+\mathbf{e}(\mathbf{t})$

$$
\begin{aligned}
& \mathbf{v}_{\alpha \beta}=\mathbf{R} \mathbf{i}_{\alpha \beta}+\mathbf{L} \frac{\mathbf{d i} \mathbf{a}_{\alpha \beta}}{\mathbf{d t}}+\mathbf{e}_{\alpha \beta} \\
& \mathbf{v}_{\mathrm{dq}}=\mathbf{e}^{-\mathrm{j} \theta} \mathbf{v}_{\alpha \beta} \\
& {\left[\begin{array}{l}
\mathbf{v}_{\mathrm{d}} \\
\mathbf{v}_{\mathrm{q}}
\end{array}\right]=\mathbf{L} \frac{\mathbf{d}}{\mathbf{d t}}\left[\begin{array}{l}
\mathbf{i}_{\mathbf{d}} \\
\mathbf{i}_{\mathbf{q}}
\end{array}\right]+\left[\begin{array}{cc}
\mathbf{R} & -\mathbf{w} \mathbf{L} \\
\mathbf{w} & \mathbf{R}
\end{array}\right]\left[\begin{array}{l}
\mathbf{i}_{\mathbf{d}} \\
\mathbf{i}_{\mathbf{q}}
\end{array}\right]+\left[\begin{array}{l}
\mathbf{e}_{\mathbf{d}} \\
\mathbf{e}_{\mathbf{q}}
\end{array}\right]}
\end{aligned}
$$

Después de transformar las corrientes al marco de referencia dq, se controla la corriente en el eje directo $\left(i_{d}\right)$ y en el eje en cuadratura $\left(\mathrm{i}_{\mathrm{q}}\right)$ utilizando un controlador Proporcional-Integral (PI). Estas salidas en sumas algebraicas con las señales de realimentación definen las señales de control, que son sintetizadas por el puente inversor. La descripción de esta técnica se ilustra en Fig. 5.

El control orientado de corriente requiere de la implementación de tres controles PI: uno en el PLL y dos para el seguimiento de las corrientes $d q$. El comportamiento del controlador orientado depende de la buena sintonización de los controles PI.

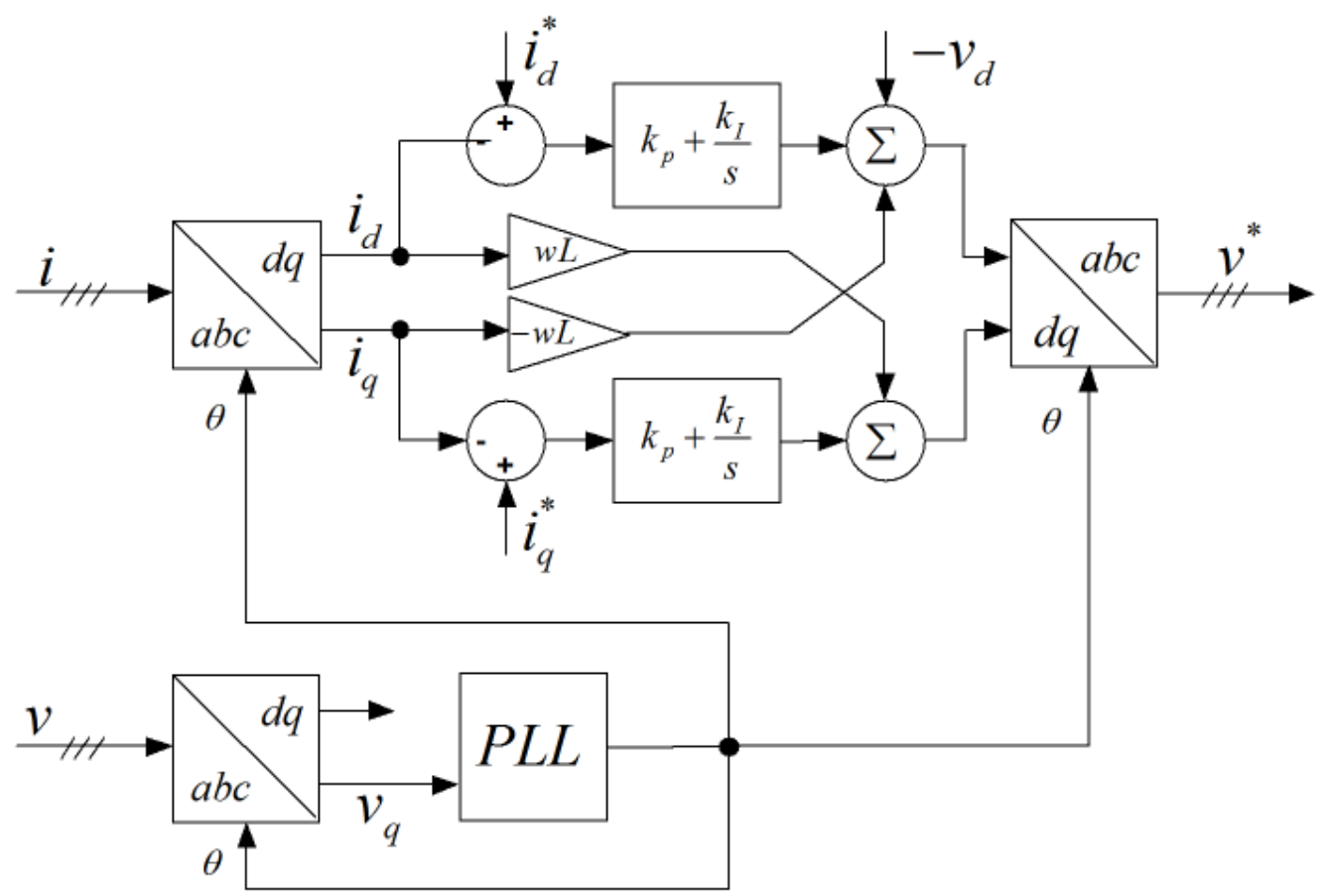

Fig. 5. Control orientado de corriente. Fuente: autores. 


\subsection{Control Predictivo}

El control predictivo basado en modelo (Model Based Predictive Control) generalizado, por la sigla MPC, es una estrategia de control que hace uso del modelo del sistema para predecir las salidas futuras y determinar la acción de control óptima aplicada en el momento presente, que minimiza el error futuro en la respuesta.

Las aplicaciones del MPC en el aprovechamiento de fuentes de energías renovables se pueden clasificar en tres grupos: control DC, control AC y optimización. Este trabajo está orientado en la conexión a red de energías renovables, siendo necesario implementar un controlador predictivo en AC. La variable de control es la corriente que se inyecta a la red, en cuyo caso se implementa un controlador predictivo de corriente.

El control predictivo propuesto, estima el comportamiento futuro y evalúa una función objetivo. El estado de conmutación del puente inversor para el intervalo siguiente es definido por aquella secuencia que minimiza el error en la corriente. La predicción se realiza a partir del modelo matemático que describe el sistema. En (5) se indica la representación en tiempo discreto que modela el convertidor VSI con conexión a red mostrado en Fig. 1, donde $\mathrm{v}(\mathrm{k})$ es el voltaje de las ramas del inversor en el instante $\mathrm{k}$ y $\mathrm{T}_{\mathrm{s}}$ es el período de muestreo. Reemplazando la derivada mediante (6) y despejando se obtiene (7), donde es posible determinar el valor predictivo de la corriente para el siguiente instante de muestreo. Para evaluar la expresión se requiere conocer valores presentes: de $\mathrm{i}(\mathrm{k}), \mathrm{v}(\mathrm{k}), \mathrm{e}(\mathrm{k})$. El valor de $\mathrm{e}(\mathrm{k})$ se obtiene por estimación utilizando muestras anteriores en (5). El valor actual estimado de la tensión de la red ê(k) se puede aproximar a su valor anterior según (8) ya que la frecuencia de la red es muy baja comparada con la frecuencia de conmutación del inversor.

$$
\begin{aligned}
& \mathbf{v}(\mathbf{k})=\mathbf{R i}(\mathbf{k})+\mathbf{L} \frac{\mathbf{d i}(\mathbf{k})}{\mathbf{d t}}+\mathbf{e}(\mathbf{k}) \\
& \frac{\mathbf{d i}(\mathbf{k})}{\mathbf{d t}}=\frac{\mathbf{i}(\mathbf{k}+1)-\mathbf{i}(\mathbf{k})}{\mathbf{T}_{\mathbf{s}}} \\
& \mathbf{i}^{\mathbf{P}}(\mathbf{k}+1)=\left[1-\frac{\mathbf{R}}{\mathbf{L}} \mathbf{T}_{\mathrm{s}}\right] \mathbf{i}(\mathbf{k})+\frac{\mathbf{T}_{\mathrm{s}}}{\mathbf{L}}[\mathbf{v}(\mathbf{k})-\mathbf{e}(\mathbf{k})] \\
& \hat{\mathbf{e}}(\mathbf{k}) \approx \hat{\mathbf{e}}(\mathbf{k}-1)=\mathbf{v}(\mathbf{k}-1)-\frac{\mathbf{L}}{\mathbf{T}_{\mathrm{s}}} \mathbf{i}(\mathbf{k})-\left[\mathbf{R}-\frac{\mathbf{L}}{\mathbf{T}_{\mathrm{s}}}\right] \mathbf{i}(\mathbf{k}-1) \\
& \mathbf{h}=\left|\mathbf{i}_{\boldsymbol{\alpha}}^{*}-\mathbf{i}_{\boldsymbol{\alpha}}^{\mathbf{P}}\right|+\left|\mathbf{i}_{\boldsymbol{\beta}}^{*}-\mathbf{i}_{\boldsymbol{\beta}}^{\mathbf{P}}\right|
\end{aligned}
$$

El control predictivo se desarrolla en coordenadas $\alpha \beta$. Inicialmente se realiza la transformación de coordenadas de las corrientes y se estima el voltaje de la red. El valor predictivo de la corriente se calcula utilizando los diferentes vectores de conmutación (ver Tabla 1), y se escoge el vector con el cual se minimiza la función objetivo $h$ en (9), donde se comparan los valores predictivos con los valores de referencia de la corriente. El algoritmo determina el vector óptimo de conmutación con el cual se obtiene la respuesta deseada en el siguiente período de muestreo. Un diagrama de bloques que describe el control predictivo se muestra en Fig. 6. 


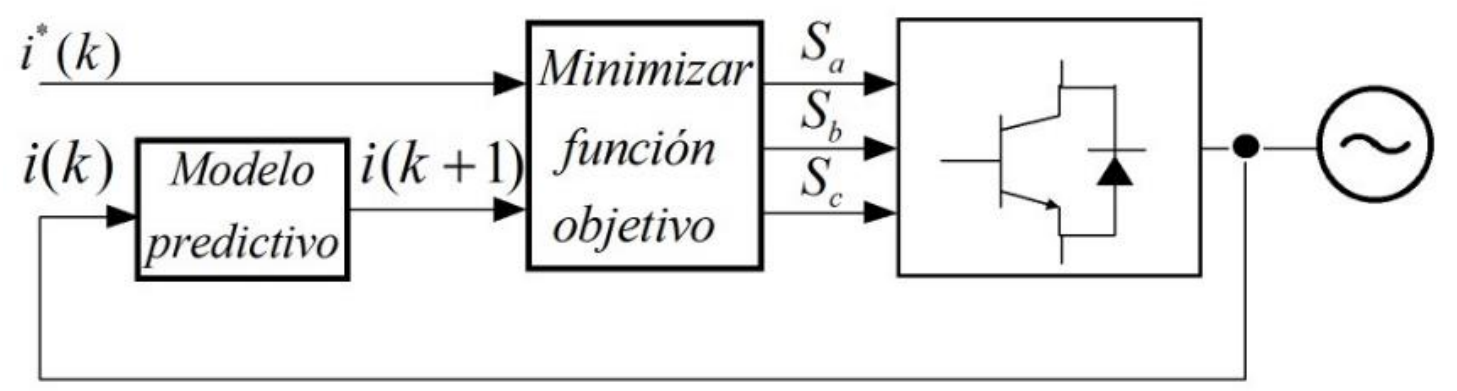

Fig. 6. Diagrama de bloques control predictivo. Fuente: autores.

\section{RESULTADOS Y DISCUSIÓN}

La técnica PWM, con la cual operan los inversores conectados a la red, ejerce un papel importante en la calidad de la energía. En su operación el inversor produce armónicos que son inyectados al sistema de potencia produciendo efectos indeseados. Luego, es pertinente utilizar una técnica PWM que produzca muy bajo contenido armónico. Inicialmente, se realiza la comparación de las técnicas SPWM, SVPWM Continuo y SVPWM Discontinuo, para determinar la técnica de menor contenido armónico que será utilizada para la implementación del control orientado de corriente. Por su parte, el controlador predictivo de corriente determina en cada instante de muestreo, la conmutación óptima aplicando la técnica SVPWM. El objetivo es comparar la mejor versión del control orientado de corriente con el controlador predictivo.

\subsection{Técnicas PWM}

Se han implementado las técnicas PWM en un circuito de prueba utilizando Simulink de Matlab (ver Fig. 7). El circuito consiste de un inversor VSI con tensión constante en el bus de continua de $440 \mathrm{~V}$ y carga RL que actúa como filtro $(\mathrm{R}=0.2 \Omega$, $\mathrm{L}=6.3 \mathrm{mH})$. Señales de referencia simétricas, sinusoidales y balanceadas de $60 \mathrm{~Hz}$. En la técnica SPWM, el índice de modulación es 0.8 y la frecuencia de la portadora $20 \mathrm{kHz}$. En la técnica SVPWM, la frecuencia de actualización de la secuencia de conmutación es también $20 \mathrm{kHz}$. El propósito es establecer condiciones equivalentes de operación para poder realizar una comparación.

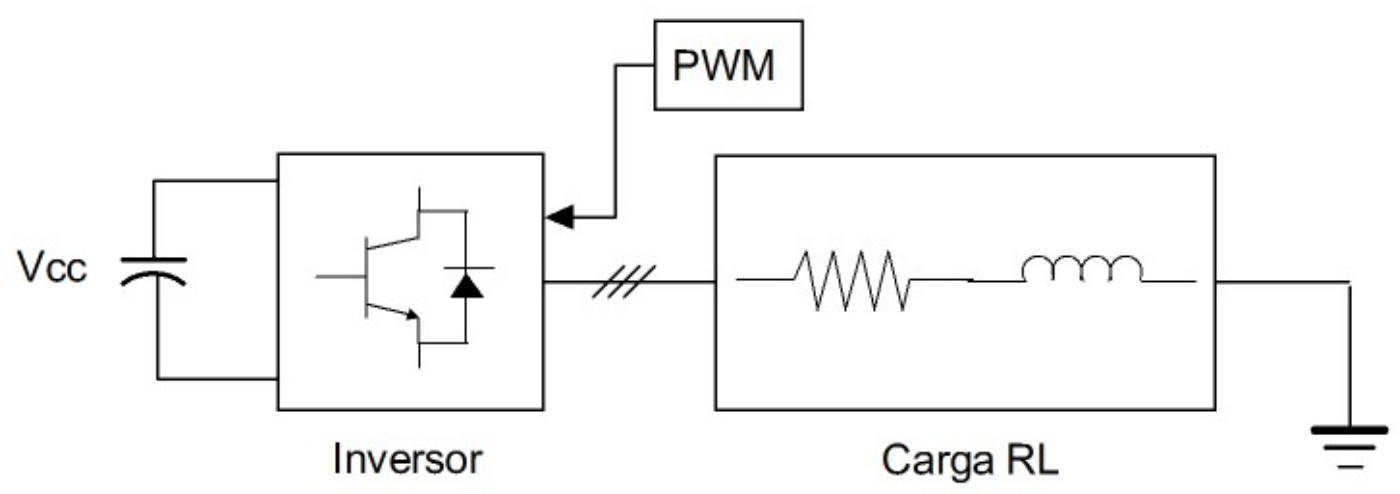

Fig. 7. Esquema simulación de técnicas PWM. Fuente: autores. 
Los resultados mostrados corresponden a una de las fases. En Fig. 8 y Fig. 9 se muestran voltajes y corrientes obtenidas en la simulación de las técnicas PWM (SPWM, SVPWM continuo y discontinuo). Las señales de corriente tienen forma sinusoidal y amplitudes diferentes. En la técnica SPWM se presenta un transitorio y la corriente alcanza un valor de régimen permanente mayor respecto a las otras técnicas. Las señales de corriente SVPWM continuo y discontinuo están en fase $\mathrm{y}$ tienen amplitudes iguales.

El análisis de armónicos se realizó utilizando la herramienta FFT de Simulink. Las gráficas se muestran en Fig. 10. En la Tabla 2, se resumen los resultados. Las componentes DC en SVPWM son mayores que en SPWM. El mismo resultado se muestra en [27].
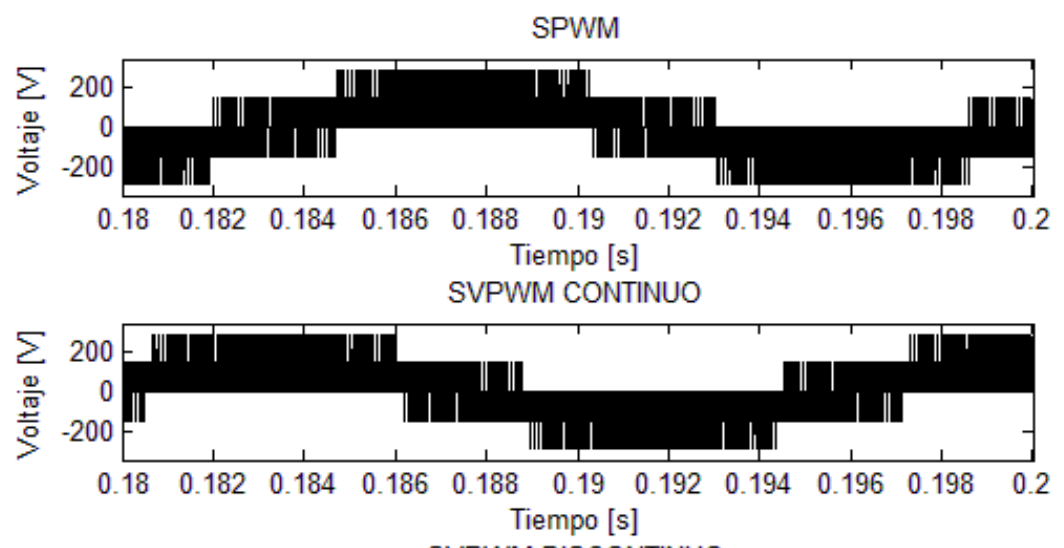

SVPWM DISCONTINUO

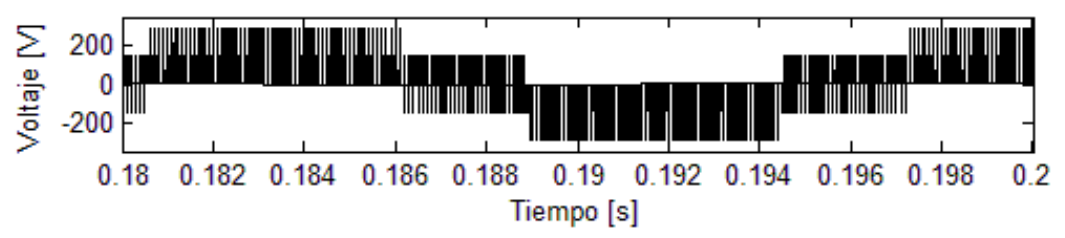

Fig. 8. Voltajes técnicos PWM. Fuente: autores.
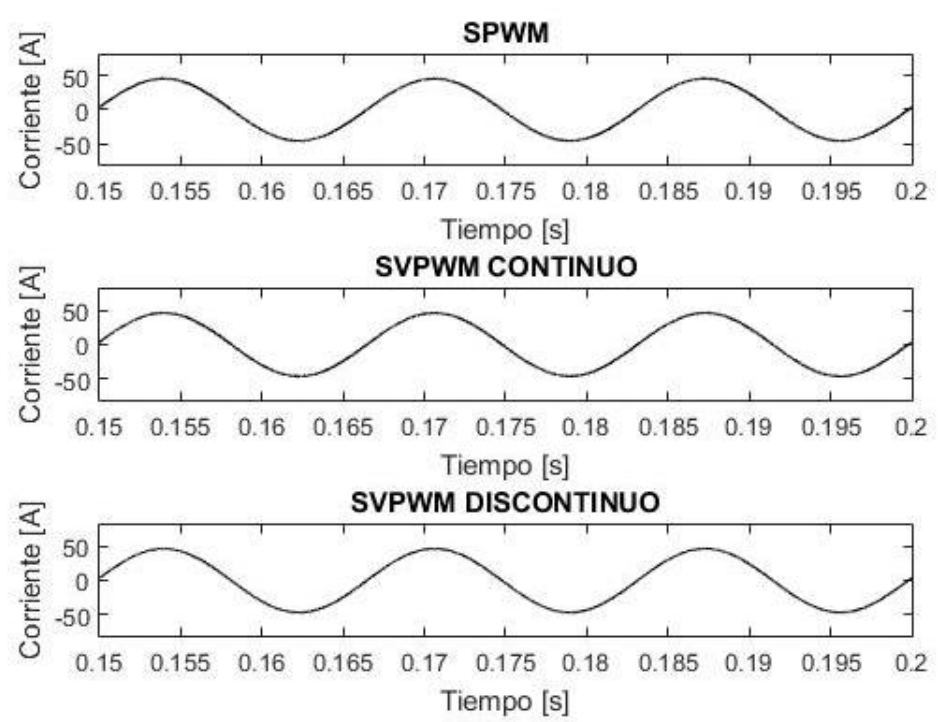

Fig. 9. Corrientes técnicas PWM. Fuente: autores. 
Análisis comparativo del control predictivo de corriente en convertidores VSI empleados en la conexión a red de energías renovables

Tabla 2. Comparación general corrientes técnicas PWM. Fuente: autores.

\begin{tabular}{lccc} 
Indicadores & SPWM [\%] & \multicolumn{2}{c}{ SVPWM [\%] } \\
& & Continuo & Discontinuo \\
\hline Componente DC [V] & 0.1175 & 0.1257 & 0.2147 \\
Fundamental [Vp] & 73.47 & 45.83 & 46.14 \\
Fundamental [Vrms] & 51.95 & 32.41 & 32.62 \\
THD [\%] & 0.32 & 0.42 & 0.66 \\
\hline
\end{tabular}

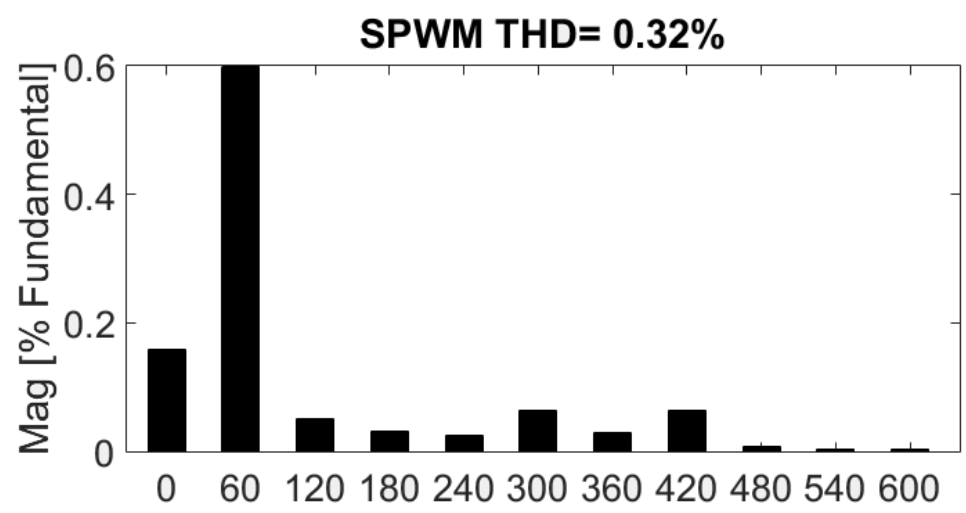

Frecuencia $[\mathrm{Hz}]$

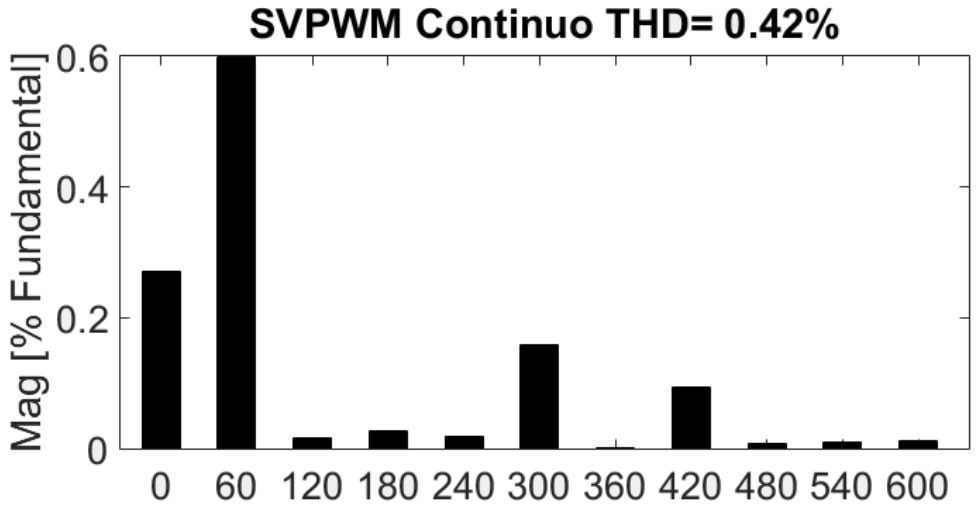

Frecuencia $[\mathrm{Hz}]$

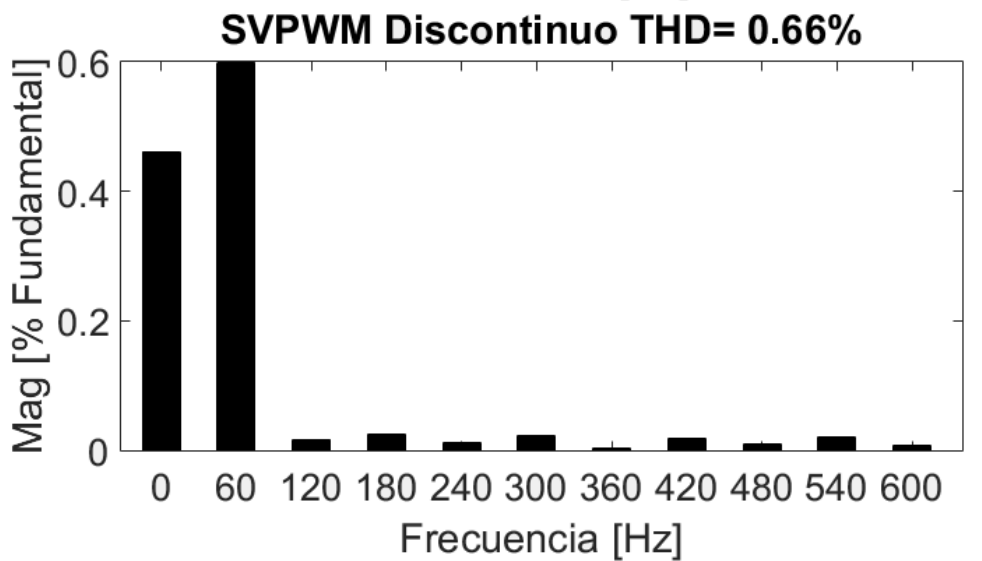

Fig. 10. Análisis de armónicos de corrientes técnicas PWM. Fuente: autores. 
Una de las variables para evaluar el buen desempeño de la modulación SPWM es el contenido armónico. Como se mencionó antes, la técnica SVPWM presenta menor índice THD comparado con técnicas basadas en portadora [21], aunque los resultados obtenidos no validan la afirmación. Es claro, que este comportamiento está definido por una adecuada selección de vectores y secuencia de conmutación [24]. Por eso es importante la optimización de esta técnica de modulación, así como se expone en [28].

\subsection{Controladores de corriente}

El circuito mostrado en Fig. 11 es el esquema de simulación implementado para la comparación de las técnicas de control de corriente. Tensión bus de continua $440 \mathrm{~V}$, impedancia de línea $\mathrm{R}=0.2 \Omega$ y $\mathrm{L}=6.3 \mathrm{mH}$, tiempo de muestreo $1 \mu \mathrm{S}$, fuente trifásica AC $220 \mathrm{~V} 60 \mathrm{~Hz}$. La corriente de referencia es de $20 \mathrm{~A}$ (valor pico).

Las siguientes son las ganancias de los controles PI en el control orientado de corriente. En el control PI del PLL, se utilizaron valores reportados en [29]: $K_{P}=$ 165.4 y $K_{I}=11834.5$. Para los controladores PI de las corrientes $d q$, las ganancias se calcularon aplicando la metodología descrita en [30], obteniendo $K_{P}=10.4$ y $K_{I}=2318.2$.
En el control orientado de corriente se utilizó la técnica SPWM que presentó menor índice THD según el análisis anterior. La modulación en el caso del control predictivo es definida mediante la secuencia de conmutación que minimiza el error de la corriente. En Fig. 12 se observa que las dos técnicas alcanzan la corriente de referencia. La corriente del control orientado presenta mayor contenido armónico. El análisis de armónicos se muestra en Fig. 13. En la Tabla 3, se resumen los resultados.

Para evaluar el comportamiento dinámico de los controladores de corriente, se implementó un cambio en la corriente de referencia. En la primera parte de la simulación la corriente de referencia es $20 \mathrm{~A}$ valor pico y para el período final es de $15 \mathrm{~A}$ valor pico. Los valores correspondientes en el eje directo son $24,4 \mathrm{~A}$ y $18,3 \mathrm{~A}$ respectivamente. En ambos casos, la corriente en cuadratura es nula. Los controladores alcanzan un valor de régimen permanente en el eje directo y en el eje en cuadratura, ver Fig. 14. En el control orientado hay una señal de ruido significativa, que es producida por la acción de los controles PI propios del controlador.

Se aprecia que el control predictivo de corriente tiene una mejor respuesta dinámica. Este controlador elimina rápidamente el error en la corriente, como se aprecia en Fig. 15.

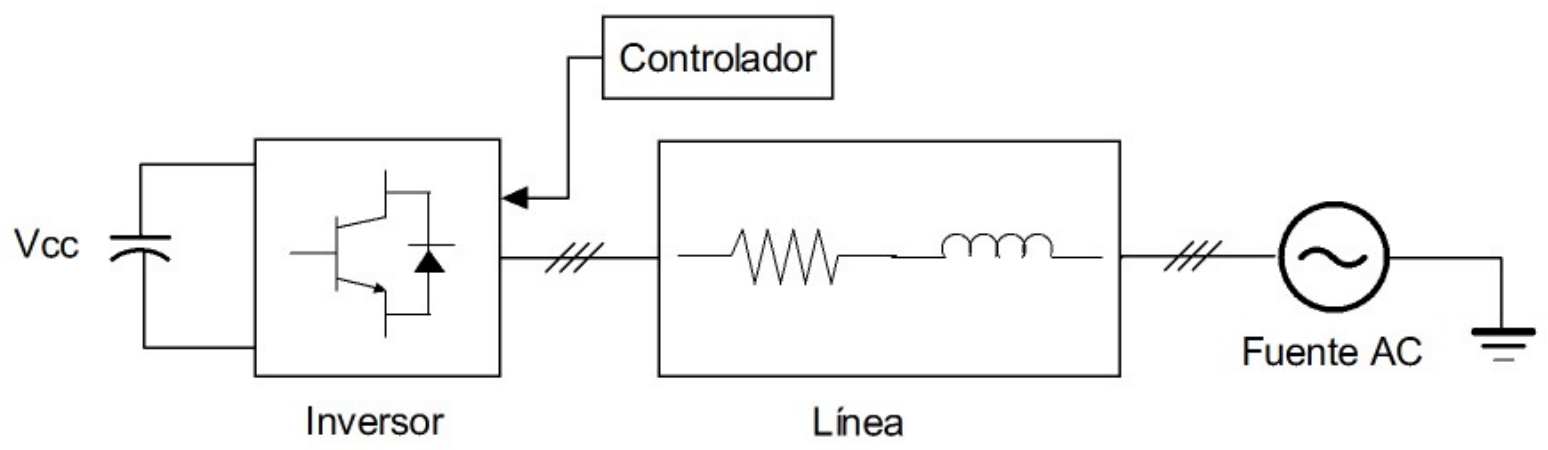

Fig. 11. Esquema simulación de técnicas de control de corriente. Fuente: autores. 

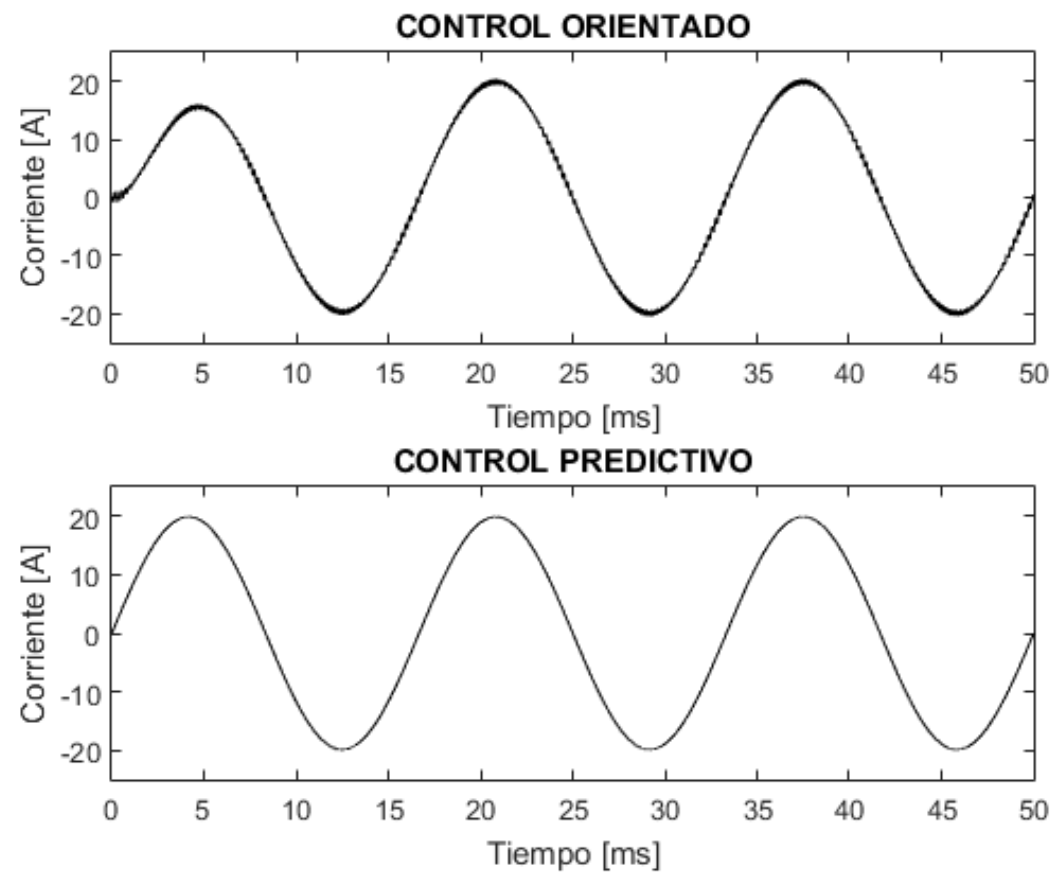

Fig. 12. Simulación técnica de control de corriente. Fuente autores.
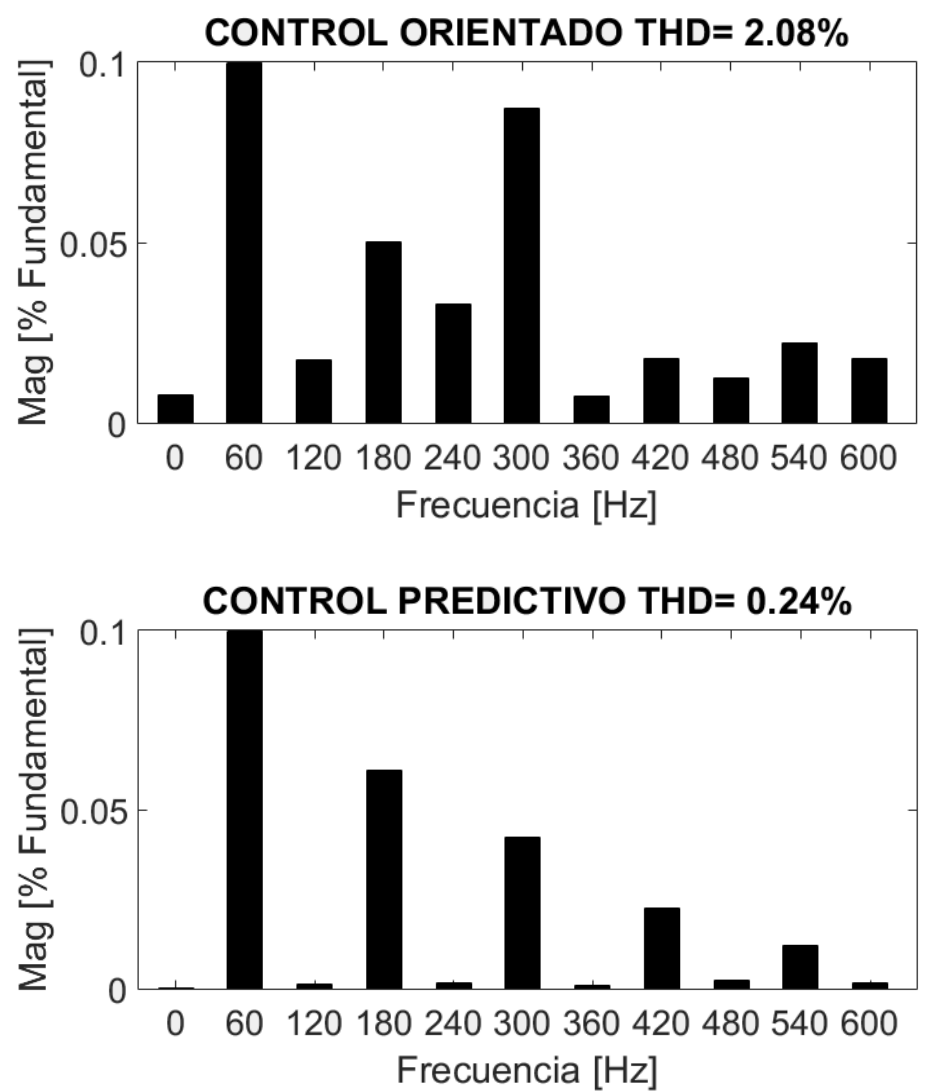

Fig. 13. Análisis de armónicos técnicas de control de corriente. Fuente: autores. 
Análisis comparativo del control predictivo de corriente en convertidores VSI empleados en la conexión a red de energías renovables
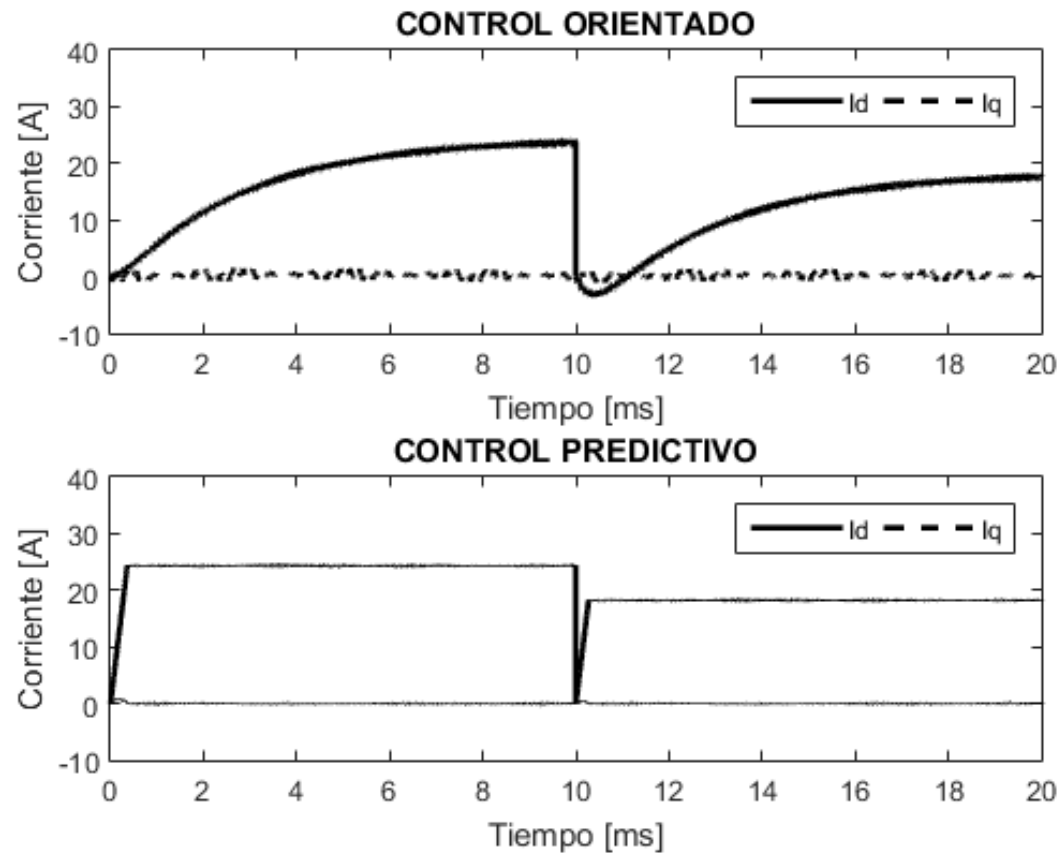

Fig. 14. Respuesta controladores de corriente en coordenadas $d q$. Fuente: autores.

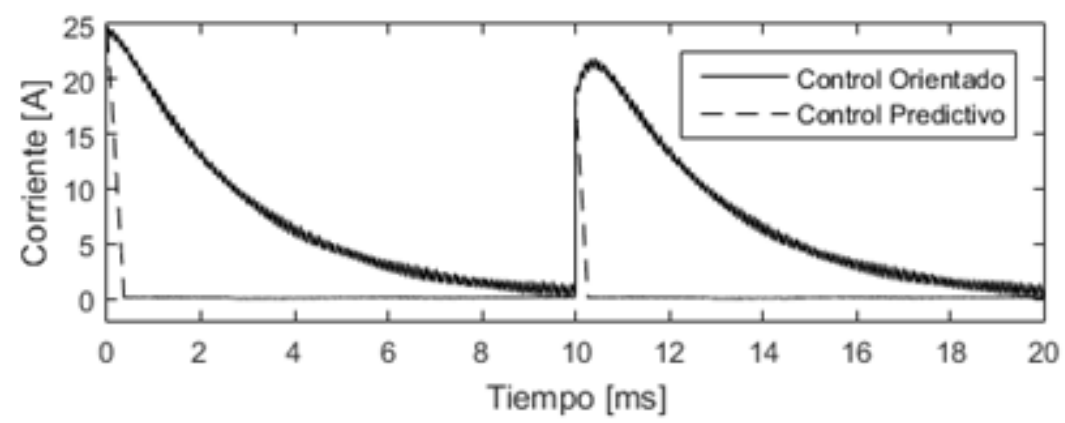

Fig. 15. Error controladores de corriente. Fuente: autores.

Tabla 3. Comparación de técnicas de control de corriente. Fuente: autores.

\begin{tabular}{ccc}
\hline Indicadores & $\begin{array}{c}\text { Control } \\
\text { orientado [\%] }\end{array}$ & $\begin{array}{c}\text { Control } \\
\text { predictivo [\%] }\end{array}$ \\
\hline Componente DC [V] & 0.00254 & 0.00023 \\
Fundamental [Vp] & 19.92 & 19.89 \\
Fundamental [Vrms] & 14.08 & 14.06 \\
THD [\%] & 2.08 & 0.24 \\
\hline
\end{tabular}


Un criterio de comparación es la característica de conmutación de los dispositivos del puente inversor. En el control orientado la conmutación depende de la técnica PWM utilizada, mientras que en el control predictivo la conmutación depende del tiempo de muestreo. La frecuencia de la portadora para la técnica SPWM en el controlador orientado de corriente es $20 \mathrm{kHz}$ y el tiempo de muestreo es $1 \mu \mathrm{S}$. En Fig. 16, se muestran los pulsos de activación del mismo dispositivo para cada controlador. Los resultados muestran una alta exigencia en la operación de los semiconductores en el control predictivo de corriente.

La respuesta de los dos controladores depende del tiempo de muestreo. En Fig. 12 , se muestran las señales de corriente obtenidas con tiempo de muestreo de $1 \mu \mathrm{S}$ y en Fig. 17, corrientes con tiempo de muestreo de $20 \mu \mathrm{S}$. A medida que aumenta el tiempo de muestreo se distorsiona la forma de onda de la corriente en cada controlador.
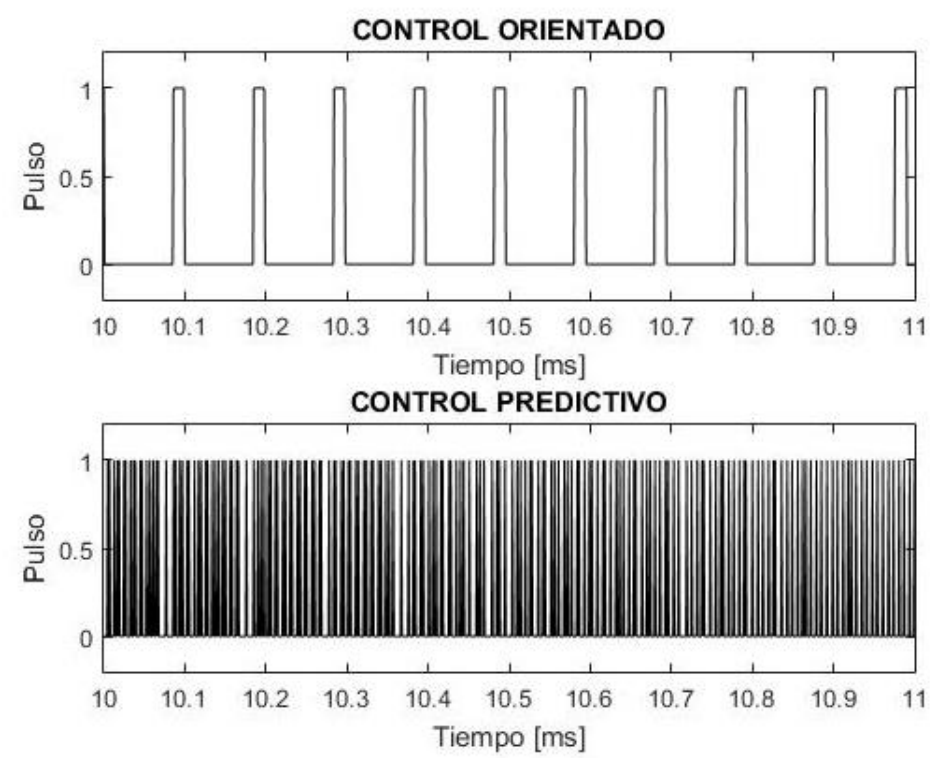

Fig. 16. Pulso de activación dispositivo puente inversor. Fuente: autores.
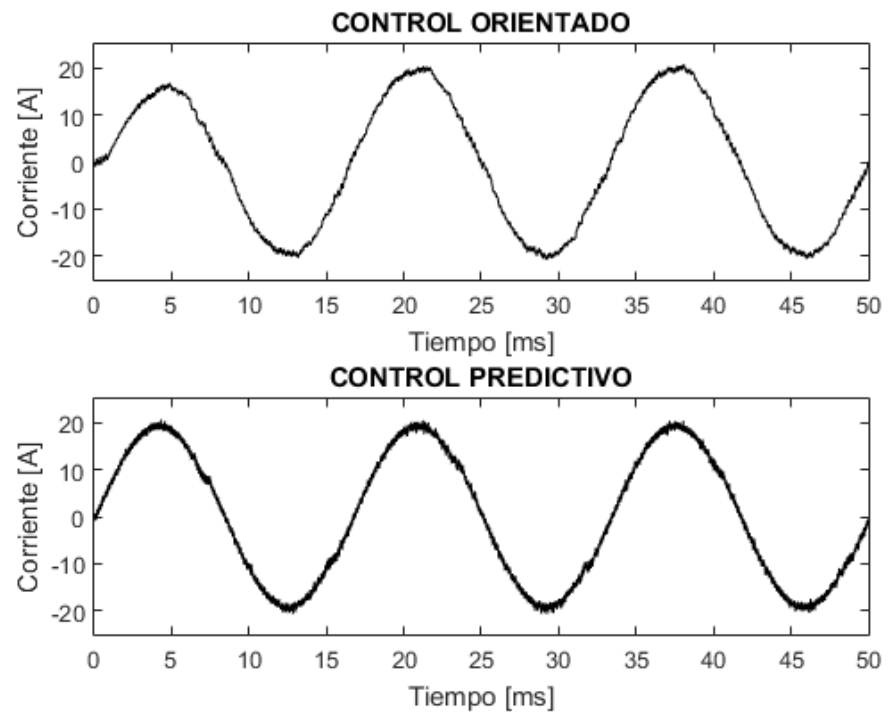

Fig. 17. Control orientado de corriente para diferentes tiempos de muestreo. Fuente: autores. 
Análisis comparativo del control predictivo de corriente en convertidores VSI empleados en la conexión a red de energías renovables

\section{CONCLUSIONES}

El control orientado de corriente requiere de tres controles PI, de tal forma que el ajuste de los parámetros es fundamental para el buen comportamiento del controlador. La sintonización es una acción adicional que hace compleja la implementación del algoritmo. En el análisis en coordenadas dq, se aprecia un ruido significativo debido a la acción de los controles PI lo que produce un alto contenido armónico. El índice THD de las corrientes obtenido para este controlador es del $2 \%$, el cual es muy superior al $0.2 \%$ obtenido con el control predictivo. El control orientado de corriente presenta una menor exigencia en la operación de los dispositivos del puente inversor, pues el número de conmutaciones es menor como se observa en Fig. 16. Esta característica tiene relación directa con la respuesta dinámica. Debido al bajo número de conmutaciones, el control orientado presenta tiempos de establecimiento altos (valores cercanos a los 10 segundos). Por su parte, el control predictivo presenta una mayor exigencia en la conmutación de los dispositivos, característica que le permite alcanzar rápidamente el estado estacionario (tiempos inferiores a 0.5 segundos).

El control predictivo define el comportamiento futuro del sistema a partir del modelo matemático. Se requiere entonces una descripción amplia del sistema para obtener buenos resultados. Una ventaja en su aplicación es que no requiere medidas de los voltajes de la red, los cuales son estimados por el propio algoritmo. En la estimación, el período de muestreo es una variable crítica que debe ser seleccionada convenientemente.

El control predictivo propuesto presenta una estructura simple en comparación con el control orientado de corriente. Es de fácil implementación, no requiere la medición de voltajes, presenta bajo contenido armónico y tiene mejor tiempo de respuesta. El control predictivo presenta un mejor com- portamiento y es la técnica de control de corriente recomendada para la conexión a red de recursos renovables.

\section{REFERENCIAS}

[1] S. Alepuz, S. Busquets-Monge, J. Bordonau, J. Gago, D. González and j. Balcells, "Interfacing renewable energy sources to the utility grid using a three-level inverter," IEEE Transactions on Industrial Electronics, vol. 53, no. 5, pp. 1504-1511, 2006.

[2] D. G. Holmes, T. A. Lipo, B. P. McGrath and W. Y Kong, "Optimized design of stationary frame three phase AC current regulators," IEEE Transactions on Power Electronics, vol. 24, no. 11, pp. 2417-2426, 2009.

[3] P. C. Sen, "Electric motor drives and control-past, present, and future," IEEE Transactions on Industrial Electronics, vol. 37, no. 6, pp. 562-575, 1990.

[4] T. M. Rowan and R. J. Kerkman, "A new synchronous current regulator and an analysis of current-regulated PWM inverters," IEEE Transactions on Industry Applications, no. 4, pp. 678-690, 1986.

[5] R. Kennel and A. Linder, "Predictive control of inverter supplied electrical drives," in Power Electronics Specialists Conference (PESC), 2000, Vol. 2, pp. 761-766.

[6] C. K. Lin, D. Y. Wu, J. W. Hu, H. C. Yu and Y. S. Lai, "Model-free predictive current control of a voltage source inverter," in International Future Energy Electronics Conference (IFEEC), 2015, pp. 1-6.

[7] B. Cao and L. Chang, "Robust predictive current control for grid-connected VSIs with compensation for time-delay effect and uncertain system disturbances," in Energy Conversion Congress and Exposition (ECCE), 2015, pp. 2698-2702.

[8] J. Rodríguez, J. Pontt, C. A. Silva, P. Correa, P. Lezana, P. Cortés and U. Ammann, "Predictive current control of a voltage source inverter," IEEE Transactions on Industrial Electronics, vol. 54, no. 1, 495-503, 2015.

[9] S. A. Larrinaga, M. A. R. Vidal, E. Oyarbide and J. R. T. Apraiz, "Predictive control strategy for DC/AC converters based on direct power control," IEEE Transactions on Industrial Electronics, vol. 54, no. 3, pp. 1261-1271, 2007.

[10] P. Cortés, L. Vattuone and J. Rodríguez, "Predictive current control with reduction of switching frequency for three phase voltage source inverters," in International Symposi- 
Análisis comparativo del control predictivo de corriente en convertidores VSI empleados en la conexión a red de energías renovables

um on Industrial Electronics (ISIE), 2011, pp. 1817-1822.

[11] Y. Luo, C. Liu and F. Yu, "Predictive current control of a new three-phase voltage source inverter with phase shift compensation," IET Electric Power Applications, vol. 11, no. 5, pp. 740-748, 2017.

[12] Y. Baek, K. J. Lee and D. S. Hyun, "Improved predictive current control for grid connected inverter applications with parameter estimation," In Industry Applications Society Annual Meeting (IAS), 2009, pp. 1-6.

[13] J. R. Fischer, S. A. González, I. Carugati, M. A. Herran, M. G. Judewicz and D. O. Carrica, "Robust predictive control of grid-tied converters based on direct power control," IEEE Transactions on Power Electronics, vol 29, no. 10, pp. 5634-5643, 2014.

[14] J. M. Geldenhuys, H. du Toit Mouton, A. Rix and T. Geyer, "Model predictive current control of a grid connected converter with LCLfilter," In Control and Modeling for Power Electronics (COMPEL), 2016, pp. 1-6.

[15] A. Iqbal, H. Abu-Rub, S. M. Ahmed, P. Cortés and J. Rodríguez, "Model predictive current control of a three-level five-phase NPC VSI using simplified computational approach," in Applied Power Electronics Conference and Exposition (APEC), 2014, pp. 2323-2330.

[16] S. Bayhan, P. Kakosimos, H. Abu-Rub and J. Rodríguez, "Model predictive control of five-level H-bridge neutral-point-clamped qZS inverter," in Annual Conference of Industrial Electronics Society (IECON), 2016, pp. 5971-5976.

[17] J. Han, T. Yang, D. Peng, T. Wang and G. Yao, "Model predictive control for asymmetrical cascaded H-Bridge multilevel gridconnected inverter with flying capacitor," in Annual Conference of Industrial Electronics Society (IECON), 2014, pp. 1611-1616.

[18] Y. Wang, W. Cong, M. Li, N. Li, M. Cao and W. Lei, "Model predictive control of modular multilevel converter with reduced computational load," in Applied Power Electronics Conference and Exposition (APEC), 2014, pp. 1776-1779.

[19] P. Liu, Y. Wang, W. Cong and W. Lei, "Grouping-sorting-optimized model predictive control for modular multilevel converter with reduced computational load," IEEE Transactions on Power Electronics, vol. 31, no.3, pp.1896-1907, 2016.
[20]

G. Gandi and J. Loncarski, "Simplified implementation of optimised carrier-based PWM in three-level inverters," Electronics Letters, vol. 50, no. 8, pp. 631-633, 2014.

[21] D. G. Holmes, "The significance of zero space vector placement for carrier-based PWM schemes," IEEE Transactions on Industry Applications, vol. 32, no. 5, pp.11221129, 1996.

[22] S. Sanusi, Z. Ibrahim, A. Jidin, M. H. Jopri, K. A. Karim and M. N. Othman, "Implementation of Space Vector Modulation for voltage source inverter," in International Conference on Electrical Machines and Systems (ICEMS), 2013 pp. 1361-1366.

[23] K. Zhou and D. Wang, "Relationship between space-vector modulation and threephase carrier-based PWM: a comprehensive analysis three-phase inverters," IEEE transactions on industrial electronics, vol. 49, no. 1, pp. 186-196, 2002.

[24] P. A. Bezerra and M. L. Heldwein, "Generation of hybrid carrier based modulation patterns," In Power Electronics Conference (COBEP), 2013, pp. 183-188.

[25] C. Schauder and H. N. Mehta, "Vector analysis and control of advanced static VAR compensators" in IEE Proceedings C (Generation, Transmission and Distribution), 1993, vol. 140, no. 4, pp. 299-306.

[26] M. A. Hassan, and M. A. Abido, "Dynamic performance improvement of an inverterbased grid-connected microgrid," in International Conference on Renewable Energy Research and Applications (ICRERA), 2013, pp. 522-527.

[27] S. Halász, "DC components and subharmonics of carrier-based PWM," in Power Electronics and Motion Control Conference (EPE/PEMC), 2012, pp. DS3c-3.

[28] F. Jenni and D. Wueest, "The optimization parameters of space vector modulation," in Fifth European Conference on Power Electronics and Applications, 1993, pp. 376-381.

[29] Q. Huang and R. Kaushik," An improved delayed signal cancellation PLL for fast grid synchronization under distorted and unbalanced grid condition," IEEE Transactions on Industry Applications, 2017.

[30] T. Zhao, Q. Zong, T. Zhang and Y. Xu, "Study of photovoltaic three-phase gridconnected inverter based on the grid voltage-oriented control," in 11th Conference on Industrial Electronics and Applications (ICIEA), 2016, pp. 2055-2060. 